\title{
CRESCIMENTO DENDRÍTICO TERCIÁRIO DA LIGA AI-3\%Cu-5,5\%Si DURANTE A SOLIDIFICAÇÃO DIRECIONAL HORIZONTAL TRANSIENTE
}

\author{
M. BRABO ${ }^{1}$, R. H. L. KIKUCHI ${ }^{2}$, A. S. BARROS ${ }^{1}$, A. L. S. MOREIRA ${ }^{1}$ e O. L. ROCHA ${ }^{2^{*}}$ \\ ${ }^{1}$ Universidade Federal do Pará - UFPA \\ ${ }^{2}$ Instituto Federal de Educação, Ciência e Tecnologia do Pará - IFPA \\ otavio.rocha@ifpa.edu.br
}

Artigo submetido em julho/2015 e aceito em julho/2015

DOI: $10.15628 /$ holos.2015.3204

\section{RESUMO}

Neste estudo, objetivando analisar as relações entre os parâmetros térmicos e os espaçamentos dendríticos terciários $\left(\lambda_{3}\right)$ a liga Al-3\%Cu-5,5\% Si foi solidificada direcionalmente sob condições de fluxo de calor transitório em um dispositivo de configuração horizontal. Velocidades de deslocamento da isoterma liquidus $\left(\mathrm{V}_{\mathrm{L}}\right)$, taxas de resfriamento $\left(\mathrm{T}_{\mathrm{R}}\right)$ e tempos locais de solidificação $\left(t_{L S}\right)$ foram experimentalmente determinados a partir das curvas de resfriamento registradas em diferentes posições do metal. Amostras transversais foram retiradas de diferentes posições do lingote e a microestrutura foi caracterizada por meio de microscopia ótica. Os resultados encontrados indicam que a variação de $\lambda_{3}$ pode ser expressa por meio de funções na forma de potência com $V_{L}, T_{R}$ e $t_{L S}$ dadas, respectivamente, por $\lambda_{3}=11\left(V_{L}\right)^{-1,1}, \lambda_{3}=44\left(T_{R}\right)^{-0,55}$ e $\lambda_{3}=$ $2,2\left(t_{L S}\right)^{0,55}$. Uma análise comparativa é realizada entre os resultados deste trabalho e aqueles apresentados na literatura para a liga investigada quando solidificada direcionalmente na configuração vertical ascendente.

PALAVRAS-CHAVE: Ligas Al-Cu-Si, Solidificação Direcional, Microestrutura Dendrítica.

\section{TERTIARY DENDRITIC GROWTH OF Al-3wt.\%Cu-5.5wt.\%Si ALLOY DURING TRANSIENT HORIZONTAL DIRECTIONAL SOLIDIFICATION}

\begin{abstract}
In this work, in order to analyzing the interconnection among solidification thermal variables and tertiary dendrite arm spacing the Al-3wt.\%Cu-5.5wt.\%Si alloy was directionally solidified under transient heat flow conditions in a directional solidification device. Tip growth rate $\left(V_{L}\right)$, cooling rate $\left(T_{R}\right)$ and solidification local time $\left(t_{L S}\right)$ were experimentally determined by cooling curves recorded during solidification process. Transverse samples were extracted from different positions of the
\end{abstract}

casting and analyzed by optical microscopy. It was observed that the tertiary spacing variation can be expressed as power law functions of $V_{L}, T_{R}$ and $t_{L S}$ given by $\lambda_{3}=11\left(\mathrm{~V}_{\mathrm{L}}\right)^{-1.1}, \lambda_{3}=44\left(\mathrm{~T}_{\mathrm{R}}\right)^{-0.55}$ and $\lambda_{3}=2.2\left(\mathrm{t}_{\mathrm{LS}}\right)^{0.55}$, respectively. A comparative analysis with other studies published in the literature for the analyzed alloy solidified in upward vertical directional condition was performed.

KEYWORDS: Al-Cu-Si alloys, Directional Solidification, Dendritic Microstructure. 


\section{INTRODUÇÃO}

Ligas de alumínio vêm recebendo enorme destaque em aplicações industriais em virtude da excepcional combinação de propriedades mecânicas, físicas e tribológicas (Rana et al., 2012). Neste contexto, ligas ternárias Al-Cu-Si têm sido amplamente utilizadas em componentes de manufatura nas indústrias automotiva e aeroespacial tendo em vista a excelente fluidez, alta resistência mecânica aliada à elevada relação resistência/peso, além de boa resistência ao desgaste (Ferreira et al., 2010; Gomes, 2012; Moutinho, 2012; Costa et al., 2015). Dentre os processos de fabricação em que essas ligas se submetem, destaca-se como principal o de fundição. Vale destacar que os produtos fundidos, depois de solidificados, podem ser caracterizados por uma imensa variedade de estruturas que possibilitam os mais diversos desempenhos mecânicos aos mesmos. Dessa forma, estabelecer correlações entre estruturas brutas de solidificação e propriedades mecânicas decorrentes é uma tarefa complexa e que se inicia pela análise dos diferentes aspectos estruturais (Garcia, 2007).

No caso de ligas metálicas que apresentam estrutura dendrítica, as propriedades dos produtos fundidos dependem fortemente dos espaçamentos entre braços dendríticos primários $\left(\lambda_{1}\right)$, secundários $\left(\lambda_{2}\right)$ e terciários $\left(\lambda_{3}\right)$. Osório et al. (2012) observaram que um arranjo dendrítico terciário bem definido fornece uma mistura eutética interdendrítica mais distribuída homogeneamente exibindo uma melhor proteção contra a corrosão em uma liga $\mathrm{Pb}-3,5 \% \mathrm{Sb}$. Da mesma forma, Spinelli et al. (2014) apresentaram um estudo experimental com ligas de soldagem $\mathrm{Sn}-0,7 \% \mathrm{Cu}-\mathrm{X} \% \mathrm{Ag}$ (onde $\mathrm{X}=1,2$ e 3 ) onde observa-se que a presença de braços dendríticos terciários promove uma melhor distribuição de compostos intermetálicos de reforço, contribuindo também para o aumento da resistência mecânica. Estes trabalhos comprovam a forte influência destes espaçamentos sobre importantes propriedades dos materiais devido à formação de uma rede dendrítica mais complexa. Convém ressaltar que uma vez os braços dendríticos terciários estejam caracterizados na estrutura dendrítica, as propriedades mecânicas devem ser correlacionadas com os mesmos (Garcia, 2007). A Figura 1 ilustra uma rede dendrítica caracterizada pela presença de braços dendríticos primários e o surgimento de braços terciários a partir de secundários.

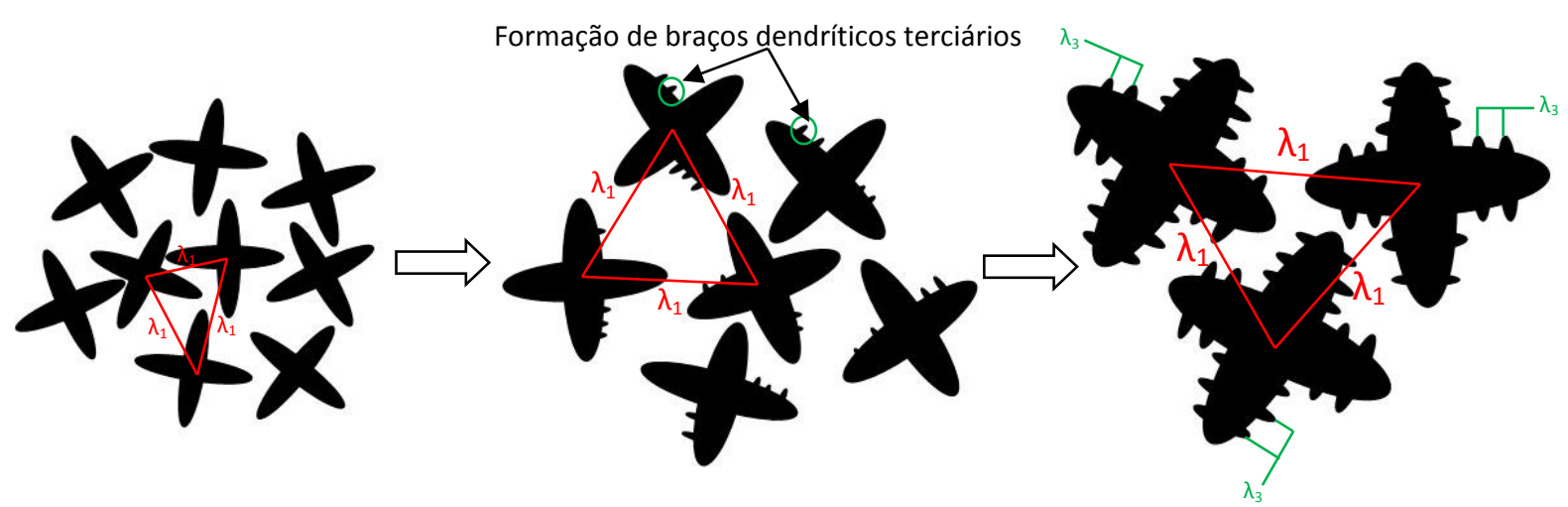

Figura 1: Representação esquemática evidenciando a presença e evolução de braços dendríticos terciários.

Estudos recentes têm analisado a significativa influência das condições térmicas envolvidas na transformação líquido/sólido, tais como velocidade de deslocamento da isoterma liquidus $\left(V_{L}\right)$, tempo local de solidificação $\left(t_{L S}\right)$ e taxa de resfriamento $\left(T_{R}\right)$, sobre os aspectos 
estruturais para diversas composições de ligas a base de alumínio (Böyük et al., 2012; Çadirli et al., 2013; Vasconcelos et al., 2014; Barros et al., 2015; Bertelli et al., 2015; Yang et al., 2015). No entanto, são raros estudos reportando os espaçamentos dendríticos terciários $\left(\lambda_{3}\right)$ em função das variáveis térmicas durante a solidificação. Gurgel (1993), a partir de experimentos realizados com ligas hipoeutéticas Al-Si em um sistema de solidificação Bridgman (Garcia 2007), determinou uma lei experimental de variação de $\lambda_{3}$ em função do tempo local de solidificação para ligas, dada por $\lambda_{3}=10\left(t_{s L}\right)^{1 / 3}$. Sá et al.(2004) observaram que os expoentes $-1,1$ e $-0,55$ caracterizam as leis experimentais de $\lambda_{3}$ em função de $V_{L}$ e $T_{R}$ para ligas hipoeutéticas Al-Cu, solidificadas direcionalmente na configuração vertical ascendente em regime transiente de extração de calor. Rosa et al. (2008) avaliaram ligas hipoeutéticas Al-Cu quando solidificadas na configuração vertical descendente e obtiveram o mesmo valor de lei de potência de $\lambda_{3}$ em função de $T_{R}$ proposto por Sá et al.(2004), mas com uma tendência de redução de cerca de 2-3 vezes de $\lambda_{3}$. Expressões de $\lambda_{3}$ em função de $V_{L}$ e $T_{R}$ para ligas multicomponentes (Al-Cu-Si) foram apresentadas pela primeira vez por Gomes (2012) e Moutinho (2012), ambos utilizando dispositivos de solidificação direcional vertical ascendente. Moutinho (2012) comparou ainda seus resultados experimentais de $\lambda_{3}$ com aqueles obtidos por Sá et al.(2004) para ligas Al-Cu,

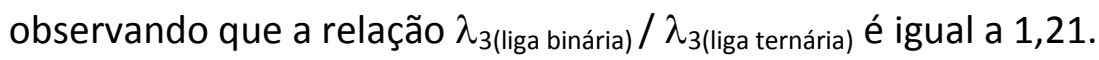

Observa-se que a maioria dos estudos sobre solidificação direcional tem se dedicado a investigar a formação de estruturas dendríticas considerando dispositivos com as configurações verticais ascendente e descendente. Recentemente, pesquisas têm sido conduzidas visando a verificação dos efeitos do vetor gravidade em relação à formação dos espaçamentos dendríticos por meio de experimentos realizados na configuração horizontal (Nogueira et al., 2012; Carvalho et al., 2013; Carvalho et al., 2014). Uma característica interessante do sistema horizontal durante a mudança de fase é o gradiente de concentração de soluto bem como os efeitos de densidade na direção vertical, pois o líquido enriquecido de soluto sempre decanta ao passo que o solvente tende a emergir devido às forças de flutuabilidade. Além disso, devido os efeitos impostos pela convecção termossolutal, sempre ocorrerá um gradiente de temperatura na direção vertical (Carvalho et al., 2013; Carvalho et al., 2014).

É importante ressaltar que pesquisas relacionadas à evolução microestrutural, utilizando sistemas de solidificação horizontal ainda são bastante raras na literatura (Atwood e Lee, 2003; Voller, 1998; Costa et al., 2015), e destacar a inexistência de correlações entre as variáveis térmicas de solidificação e o comportamento de $\lambda_{3}$ para ligas ternárias nesta configuração. Considerando o exposto, o principal objetivo deste estudo é analisar a correlação entre os parâmetros térmicos e os espaçamentos dendríticos terciários $\left(\lambda_{3}\right)$ da liga $\mathrm{Al}-3 \% \mathrm{Cu}-5,5 \% \mathrm{Si}$ quando solidificada direcionalmente em um sistema de configuração horizontal refrigerado a água, sob condições transientes de fluxo de calor. Um estudo comparativo entre os resultados obtidos neste trabalho e valores apresentados na literatura para $\lambda_{3}$ da liga estudada, quando solidificada direcionalmente no sistema vertical ascendente sob as mesmas condições assumidas neste trabalho, também é realizado.

\section{METODOLOGIA}

O dispositivo de solidificação utilizado neste estudo é apresentado na Figura 2. Este dispositivo foi projetado de tal modo que a extração de calor fosse realizada somente por uma 
das paredes laterais (refrigerada a água) da lingoteira promovendo a solidificação direcional horizontal. As resistências elétricas do forno tiveram sua temperatura controlada a fim de permitir a obtenção dos níveis de superaquecimentos desejados no metal líquido. A coquilha de aço inox utilizada apresenta $160 \mathrm{~mm}$ de comprimento, $60 \mathrm{~mm}$ de largura, $60 \mathrm{~mm}$ de altura e 3 $\mathrm{mm}$ de espessura. Suas superfícies laterais internas foram revestidas com camadas de alumina e a parte superior da mesma foi isolada com material cerâmico refratário a fim de evitar perdas de calor permitindo, dessa forma, a direcionalidade desejada para o fluxo de calor.

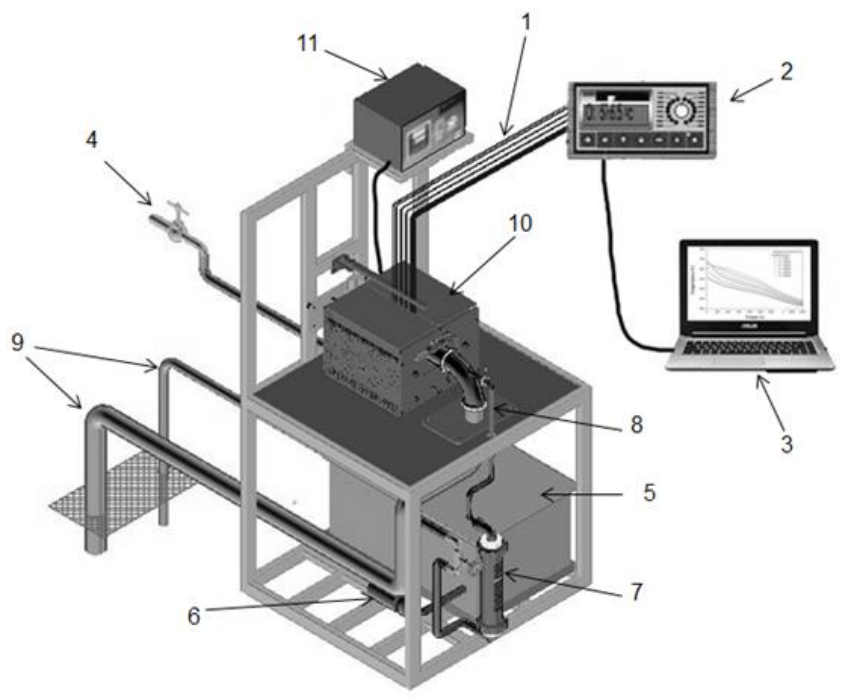

a)

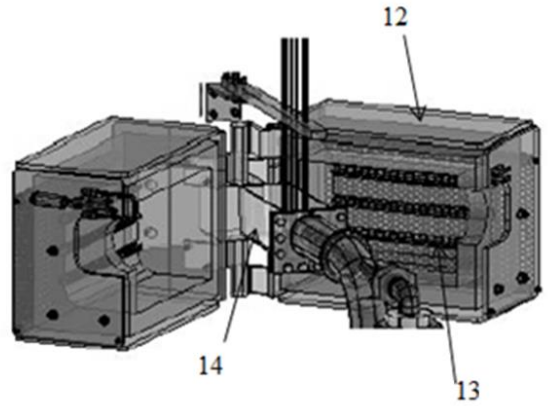

b)

Figura 2: Representação esquemática do dispositivo de solidificação direcional horizontal: 1- termopares , 2FieldLogger, 3- computador de aquisição de dados, 4- alimentação de água, 5- recipiente de água, 6- bomba de água, 7- rotâmetro, 8- entrada de água, 9- saída de água, 10- dispositivo de solidificação direcional, 11controlador de temperatura ; b) visão da parte interna do dispositivo de solidificação: 12-isolante térmico cerâmico, 13- aquecedores elétricos, 14- lingoteira retangular (de aço inoxidável).

As temperaturas no metal fundido foram monitoradas durante a solidificação por termopares do tipo $\mathrm{K}$ (Chromel-Alumel) posicionados a 5, 15, 30, 50, 70 e $90 \mathrm{~mm}$ em relação à câmara de refrigeração. Estes termopares foram conectados a um registrador de dados interligado a um computador para acompanhar, registrar e armazenar os dados obtidos durante o processo de solidificação, além de possibilitar um monitoramento on-line dos dados medidos. As composições químicas dos metais utilizados na preparação da liga analisada são apresentadas na Tabela 1.

Tabela 1: Composição química (\% em peso) dos metais utilizados na preparação da liga Al-5,5\%Si-3\%Cu.

\begin{tabular}{c|c|c|c|c|c|c|c|c|c}
\hline Metal & $\mathrm{Al}$ & $\mathrm{Fe}$ & $\mathrm{Ni}$ & $\mathrm{Si}$ & $\mathrm{Ca}$ & $\mathrm{Ti}$ & $\mathrm{Zn}$ & $\mathrm{Ga}$ & $\mathrm{Cu}$ \\
\hline $\mathrm{Al}$ & 99,7 & 0,176 & 0,006 & 0,062 & - & 0,009 & 0,007 & 0,012 & - \\
$\mathrm{Si}$ & 0,1094 & 0,3164 & 0,0102 & 99,596 & 0,0214 & 0,0455 & - & - & - \\
$\mathrm{Cu}$ & 0,0951 & - & - & - & - & - & - & - & 99,859 \\
\hline
\end{tabular}

O processo de solidificação consistiu no vazamento da liga Al-5,5\%Si-3\%Cu no interior da coquilha, localizada a parte superior do dispositivo, devidamente aquecida por resistências elétricas. Atingido o nível de superaquecimento de $10 \%$ acima da temperatura liquidus, o qual normalmente representa o grau de superaquecimento adotado na indústria de fundição, as resistências elétricas do dispositivo foram desligadas, o sistema de resfriamento foi acionado e os jatos do fluido refrigerante induziram uma extração de calor longitudinal. 
Para caracterização e quantificação da estrutura dendrítica foram retirados transversalmente ao longo do comprimento longitudinal do lingote nove corpos de prova, nas posições correspondentes a 2, 4, 6, 8, 10, 15, 20 e $30 \mathrm{~mm}$ em relação à interface metal/molde. As amostras foram lixadas e polidas com pasta de diamante de $6 \mu \mathrm{m}$ até $1 \mu \mathrm{m}$. Em seguida, foi realizado o ataque químico com solução de $5 \% \mathrm{NaOH}$ em água durante 15 segundos para revelar a microestrutura. As imagens foram obtidas em um microscópio ótico Olympus modelo BX51, sendo as medições de $\lambda_{3}$ posteriormente realizadas utilizando-se o software Image Tool (IT). 0 método utilizado para quantificar os valores desses espaçamentos foi descrito anteriormente na literatura (Sá et al., 2004; Rosa et al., 2008; Dias et al., 2015). Neste método, considera-se o valor de $\lambda_{3}$ pela média das distâncias entre os braços adjacentes ao longo do comprimento " $\mathrm{n}$ " sobre a seção transversal do lingote, aproximadamente no mesmo sentido da ramificação dendrítica primária sendo que, ao final, são obtidos a média dos valores e o intervalo de dispersão.

\section{RESULTADOS E DISCUSSÕES}

$\mathrm{Na}$ Figura 3a são apresentadas as curvas de resfriamento para os seis termopares inseridos no metal nas posições anteriormente descritas em metodologia. Estas curvas foram utilizadas para calcular a posição da isoterma liquidus em função do tempo. A função $P=f(t)$ foi definida experimentalmente pela interseção da reta equivalente à temperatura liquidus $\left(T_{L}\right)$ da liga com o perfil térmico correspondente à posição de cada termopar. Os resultados dos pares ordenados $(P, t)$ obtidos a partir do procedimento em questão possibilitam que seja plotado um gráfico experimental da posição da isoterma liquidus com o tempo, conforme mostrado na Figura 3b.

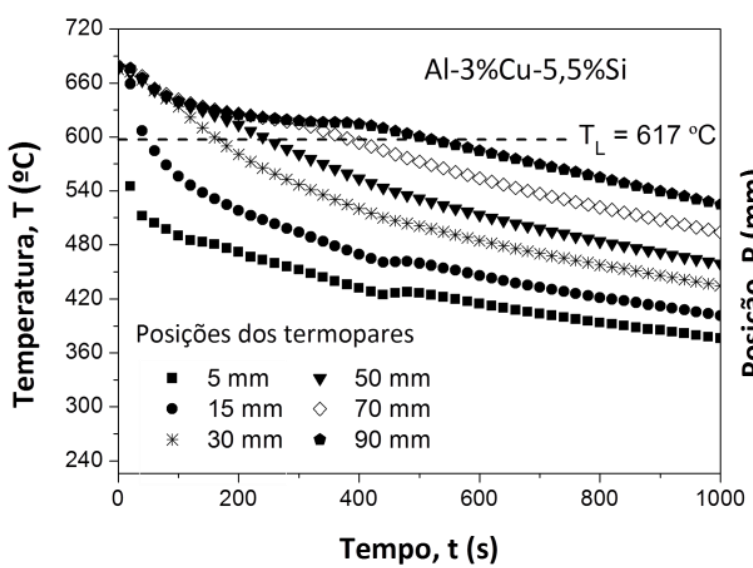

a)

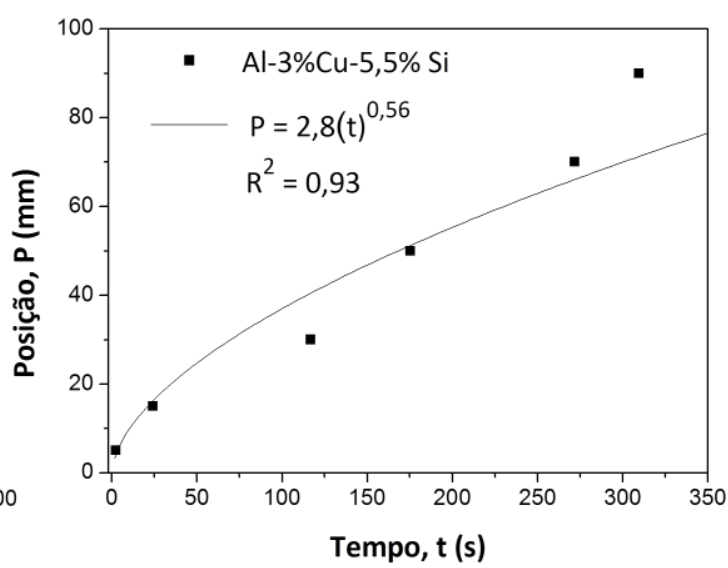

b)

Figura 3: a) Curvas experimentais de resfriamento; b) Posição da isoterma liquidus a partir da interface metal/molde em função do tempo.

A partir da derivada da função potência da posição com relação ao tempo, ou seja, $\mathrm{dP} / \mathrm{dt}$, são obtidos os respectivos valores experimentais das velocidades de deslocamento da isoterma liquidus $\left(\mathrm{V}_{\mathrm{L}}\right)$ a partir da interface metal/molde em função do tempo, o que corresponde à passagem da frente líquida por cada termopar. A Figura 4a apresenta os resultados obtidos, e é possível observar a diminuição progressiva da velocidade com o avanço da solidificação. $\mathrm{Na}$ determinação dos valores das taxas de resfriamento $\left(T_{R}\right)$ foi utilizada uma metodologia 
apresentada recentemente na literatura (Nogueira et al., 2012; Carvalho et al., 2013; Carvalho et al., 2014; Vasconcelos et al., 2014, Barros et al., 2015). O método consiste em calcular a taxa de resfriamento para cada posição dos termopares a partir das interseções da reta da temperatura liquidus com os perfis térmicos equivalentes a cada posição dos termopares, através do resultado da leitura direta do quociente das temperaturas imediatamente antes e depois da temperatura liquidus e dos tempos correspondentes, isto é, $T_{R}=\Delta T / \Delta t$. Assim, com base nos pontos experimentais foram geradas funções potência da taxa de resfriamento em função da posição cujos resultados encontram-se indicados na Figura $4 b$.

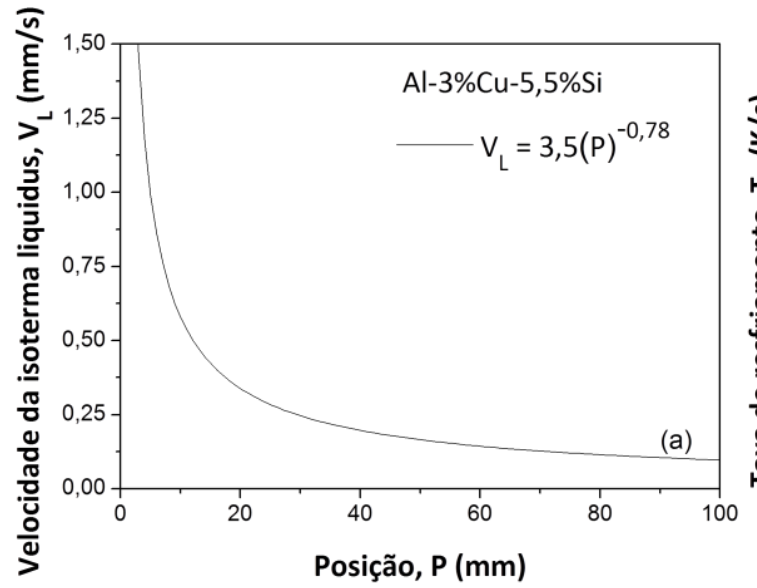

a)

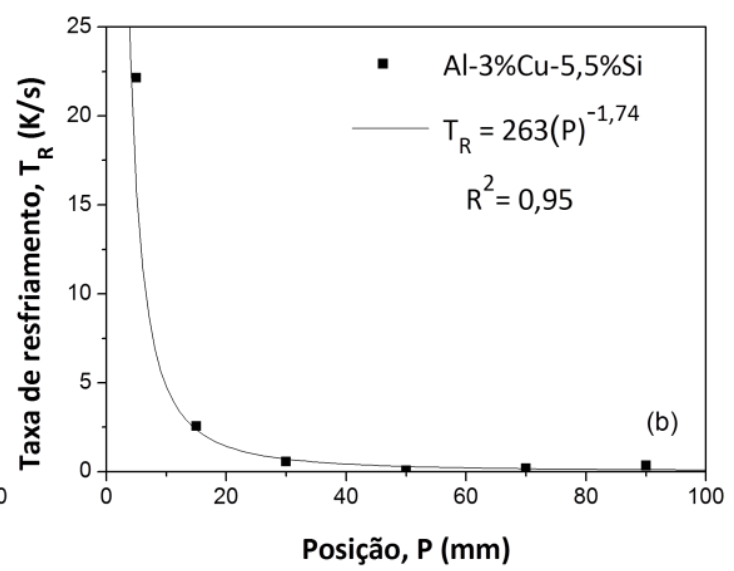

b)

Figura 4: a) Velocidades de deslocamento da isoterma liquidus e b) Taxas de resfriamento em função da posição a partir da interface metal/molde.

O tempo local de solidificação, definido como o intervalo de tempo entre a passagem da ponta e a raiz da dendrita, para o caso de solidificação direcional, foi determinado experimentalmente para cada termopar identificando-se o instante de tempo em que as posições das isotermas liquidus e solidus passam por um determinado termopar, sendo os resultados encontrados apresentados na Figura 5.

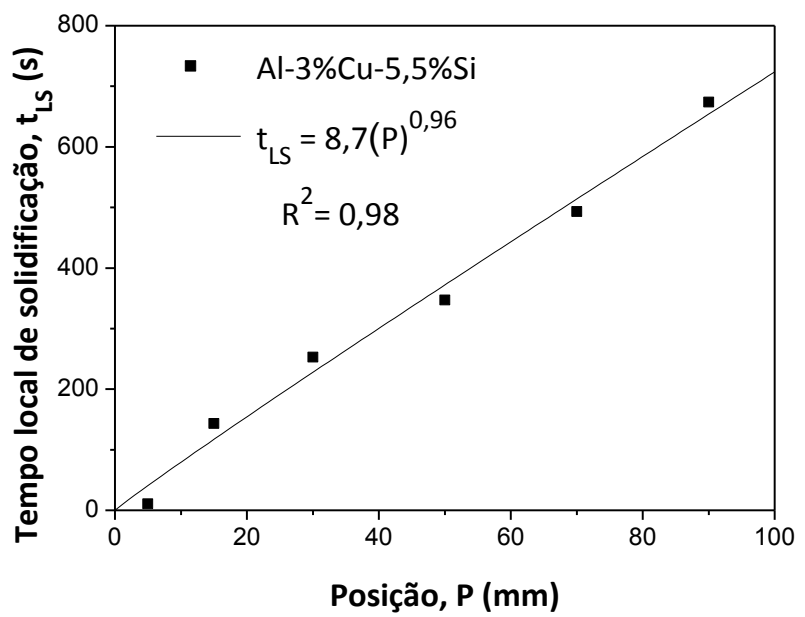

Figura 5: Tempos locais de solidificação em função da posição a partir da interface metal/molde. 
Observa-se uma diminuição da velocidade de deslocamento da isoterma liquidus e da taxa de resfriamento com a posição da isoterma liquidus, ao passo que os tempos locais de solidificação aumentam com o avanço da solidificação, isto é, quanto mais afastados da base refrigerada menores são os valores de $V_{L}$ e $T_{R}$ enquanto $t_{L S}$ são maiores. Tal fato pode ser explicado pela elevação da resistência térmica à extração de calor por condução ao longo do processo de solidificação, devido ao aumento da camada solidificada.

A fim de representar e comparar a evolução das microestruturas dendríticas bem como as condições locais de solidificação, na Figura 6 são apresentadas micrografias características da liga Al-3\%Cu-5,5\%Si e os correspondentes valores de $V_{L}, T_{R}$, $t_{L S}$ e $\lambda_{3}$ em três diferentes posições do lingote.

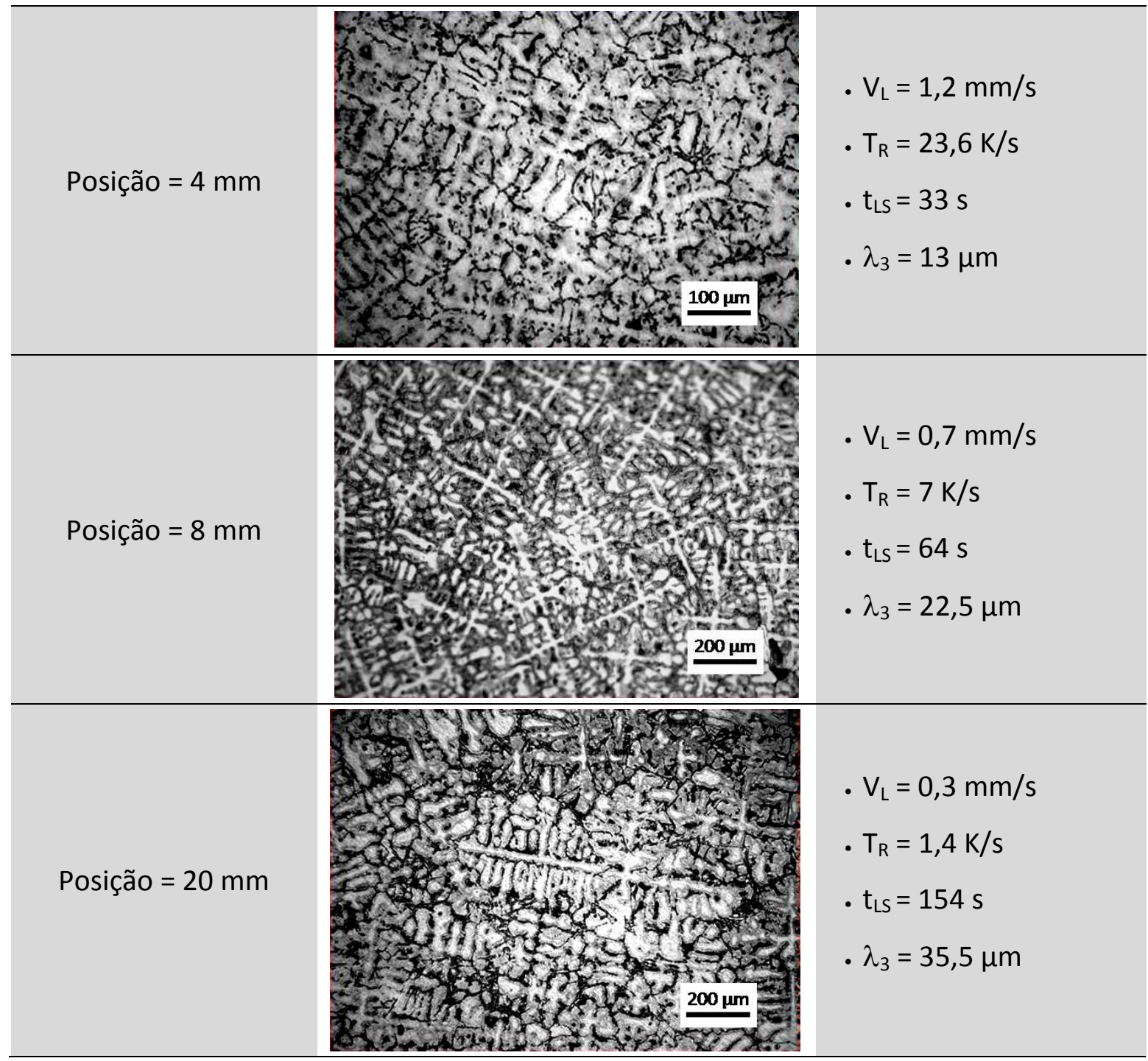

Figura 6: Microestruturas dendríticas obtidas para as posições de 4, 8 e $20 \mathrm{~mm}$ a partir da interface de resfriamento.

As Figuras 7a, 7b, 7c e 7d apresentam os resultados experimentais das medições dos espaçamentos dendríticos terciários à medida que a solidificação evolui. As linhas equivalem às equações experimentais obtidas e representam, respectivamente, as leis de crescimento desses espaçamentos com a posição, velocidade, taxa de resfriamento e tempo local de solidificação. Observa-se, em todos os casos, um excelente coeficiente de correlação $\left(R^{2}>0,7\right)$ entre os pontos 
e as curvas correspondentes. Pode ser observado também que os expoentes -1,1 e -0,55 caracterizam as leis experimentais de $\lambda_{3}$ em função de $V_{L}$ e $T_{R}$, respectivamente. Tais expoentes são os mesmos encontrados por Sá et al. (2004) e Rosa et al. (2008) para ligas Al-Cu, bem como Gomes (2012) e Moutinho (2012) para as ligas ternárias Al-Cu-Si.

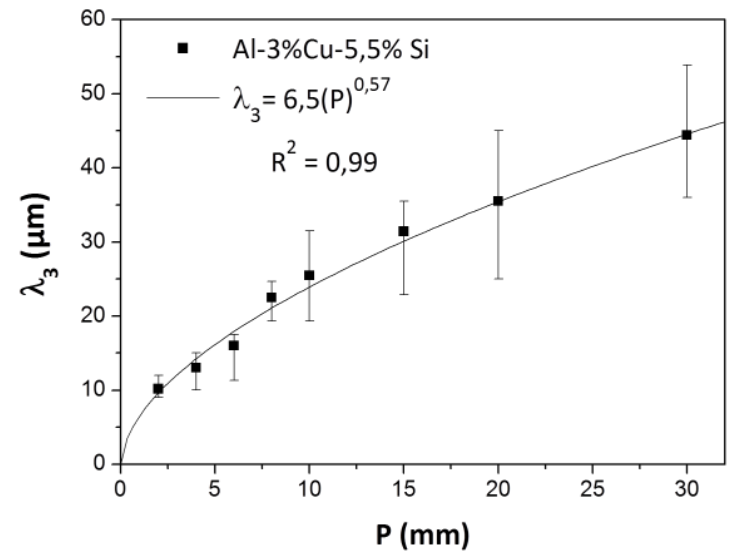

a)

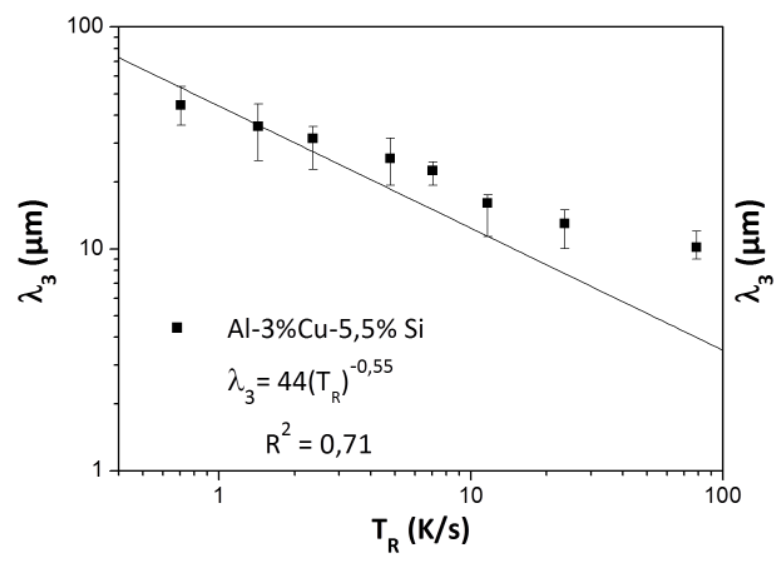

c)

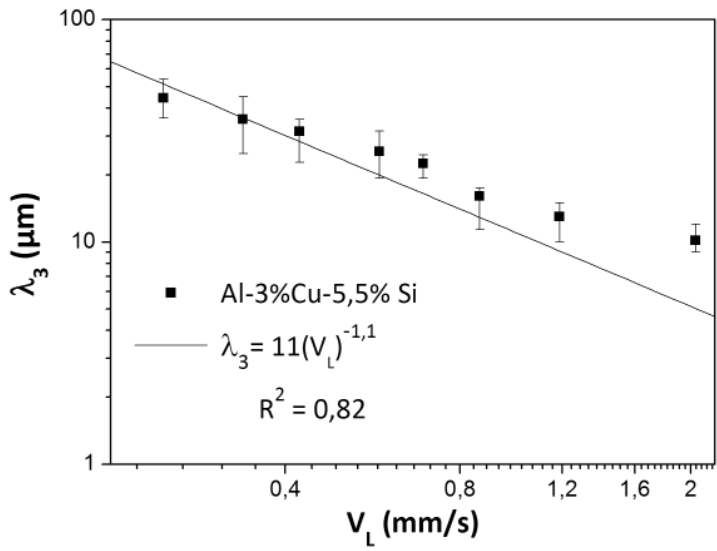

b)

Figura 7: Espaçamentos dendríticos terciários em função de - a) Posição; b) Velocidade de deslocamento da isoterma liquidus; c) Taxa de resfriamento; d) Tempo local de solidificação.

Na Tabela 2 é apresentada uma análise comparativa entre os resultados obtidos neste trabalho com os da literatura para ligas à base de alumínio, desenvolvidos nos sistemas de solidificação direcional vertical ascendente e descendente, todos sob condições transientes de extração de calor. De maneira geral, verifica-se, pelos resultados mostrados na Tabela 2, que a tanto a adição de silício nas ligas Al-Cu quanto a direção de crescimento (horizontal ou vertical) não afetam a lei de crescimento de braços dendríticos terciários em função da taxa de resfriamento, isto é, para todos os casos a lei de potência é mantida e representada matematicamente pela expressão $\lambda_{3}=K\left(T_{R}\right)^{-0,55}$. Por outro lado, evidencia-se pela Tabela 2 que a direção de crescimento vertical descendente da solidificação afeta os valores da constante $K$, observando-se valores desta constante iguais a $\mathrm{K}=18$ e 22 para as ligas Al-Cu do trabalho de Rosa et al. [16], os quais são aproximadamente 2-3 vezes menores que os valores verificados paraas demais ligas binárias e ternárias. 
Tabela 2: Leis experimentais para previsão dos espaçamentos dendríticos terciários em ligas dos sistemas Al-Cu e Al-Cu-Si solidificadas direcionalmente.

\begin{tabular}{|c|c|c|c|}
\hline Autor & Configuração & Liga & Lei \\
\hline Sá et al. [15] & Vertical ascendente & $\begin{array}{c}\text { Al-5\%Cu } \\
\text { Al-8\%Cu } \\
\text { Al-10\%Cu } \\
\text { Al-15\%Cu }\end{array}$ & $\lambda_{3}=50\left(T_{R}\right)^{-0,55}$ \\
\hline \multirow{2}{*}{ Rosa et al. [16] } & \multirow{2}{*}{ Vertical descendente } & $\mathrm{Al}-5 \% \mathrm{Cu}$ & $\lambda_{3}=18\left(T_{R}\right)^{-0,55}$ \\
\hline & & $\mathrm{Al}-8 \% \mathrm{Cu}$ & $\lambda_{3}=22\left(T_{R}\right)^{-0,55}$ \\
\hline Moutinho [4] & Vertical ascendente & $\begin{array}{l}\text { Al-6\%Cu-1\%Si } \\
\text { Al-6\%Cu-4\%Si }\end{array}$ & $\lambda_{3}=41,2\left(T_{R}\right)^{-0,55}$ \\
\hline Gomes [3] & Vertical ascendente & $\begin{array}{c}\text { Al-3\%Cu-5,5\%Si } \\
\text { Al-3\%Cu-9\%Si }\end{array}$ & $\lambda_{3}=50\left(T_{R}\right)^{-0,55}$ \\
\hline Este trabalho & Horizontal & Al-3\%Cu-5,5\%Si & $\lambda_{3}=44\left(T_{R}\right)^{-0,55}$ \\
\hline
\end{tabular}

A Figura 8 apresenta um gráfico comparativo entre os resultados deste trabalho com a lei de crescimento proposta por Gomes (2012). Observa-se, portanto, que o valor da constante $\mathrm{K}=44$, encontrada para a lei de crescimento de $\lambda_{3}=f\left(T_{R}\right)$ deste trabalho, é muito próximo daquele encontrado por Gomes (2012), mostrando que os efeitos impostos pela convecção termosolutal devido, respectivamente, ao gradiente de temperatura provocado pelo superaquecimento e a movimentação de soluto no liquido, características da solidificação direcional horizontal, praticamente nada afetam nos espaçamentos dendríticos terciários. A partir do método Global Fitting do software OriginPro, em que uma única curva é para vários pontos amostrais, observouse que uma única lei experimental é capaz de representar o crescimento de $\lambda_{3}$ em função de $T_{R}$, representada pela equação $\lambda_{3}=48\left(T_{R}\right)^{-0,55}$, para as configurações vertical ascendente (Gomes 2012) e horizontal com boa aproximação da curva para os pontos experimentais $\left(R^{2}=0,90\right)$.

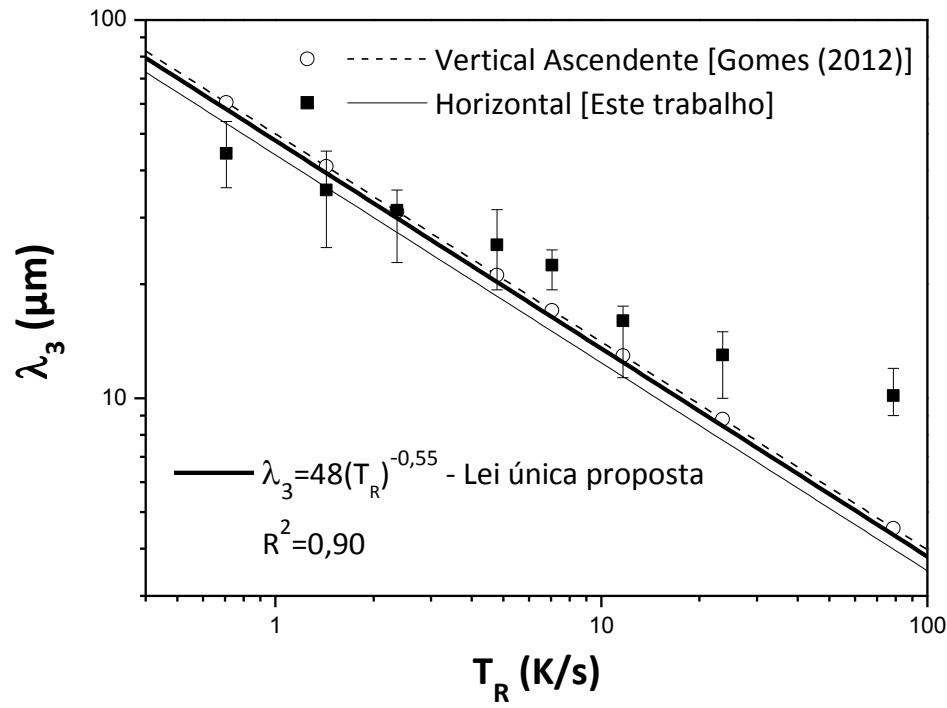

Figura 8: Influência do sentido de crescimento do sólido nos espaçamentos dendríticos terciários. 


\section{CONCLUSÕES}

O estudo desenvolvido neste trabalho sobre os espaçamentos dendríticos terciários da liga Al-3\%Cu-5,5\%Si solidificada direcionalmente em um sistema refrigerado a água de configuração horizontal, sob condições transientes de extração de calor, permite que sejam extraídas as seguintes conclusões:

- Os valores experimentais de $V_{L}$ e $T_{R}$ diminuem com o avanço da isoterma liquidus, isto é, quanto mais próximo da superfície de extração de calor, maiores valores são encontrados para esses parâmetros, podendo ser representados por equações na forma de potência em função da posição dadas, respectivamente, por $V_{L}=3,5(P)^{-0,78}$ e $T_{R}=263(P)^{-1,74}$.

- Os espaçamentos entre braços dendríticos terciários aumentam progressivamente da interface metal/molde para o interior do lingote, em virtude da diminuição gradativa dos valores de velocidades e taxas de resfriamento promovida pela formação do metal sólido.

- A evolução dos espaçamentos dendríticos terciários para a liga Al-3\%Cu-5,5\%Si solidificada nas condições assumidas neste trabalho pode ser representada por leis experimentais de crescimento em função de $V_{L}, T_{R}$ e $t_{L S}$, dadas, respectivamente, por $\lambda_{3}=$ $11\left(\mathrm{~V}_{\mathrm{L}}\right)^{-1,1}, \lambda_{3}=44\left(\mathrm{~T}_{\mathrm{R}}\right)^{-0,55}$ e $\lambda_{3}=2,2\left(\mathrm{t}_{\mathrm{LS}}\right)^{0,55}$.

- A lei experimental $\lambda_{3}=f\left(T_{R}\right)$ obtida neste estudo foi muito próxima daquela encontrada para a liga Al-3\%Cu-5,5\%Si quando solidificada na direção vertical ascendente, indicando que a convecção termossolutal parece não influenciar os valores dos espaçamentos dendríticos terciários da liga estudada quando solidificada direcionalmente da configuração horizontal, sendo proposta uma única lei de crescimento dendrítico terciário para representar a variação destes espaçamentos em função da taxa de resfriamento para as duas configurações, dada por: $\lambda_{3}=48\left(T_{R}\right)^{-0,55}$.

\section{AGRADECIMENTOS}

Os autores agradecem ao Conselho Nacional de Desenvolvimento Científico e Tecnológico (CNPq, Brasil), Instituto Federal de Educação, Ciência e Tecnologia do Pará (IFPA) e a Universidade Federal do Pará, pelo apoio financeiro e na concessão de bolsas de Iniciação Científica e de Desenvolvimento Tecnológico para os alunos do IFPA.

\section{REFERÊNCIAS BIBLIOGRÁFICAS}

1. ATWOOD, R.C., LEE, P.D. Simulation of the three-dimensional morphology of solidification porosity in an aluminium-silicon alloy. Acta Materialia, v.51, p.5447-5466, 2003.

2. BARROS, A.S., MAGNO, I.A.B., SILVA, F.P., MOTA, C.A., MOREIRA, A.L.S., SILVA, M.A.P.S., ROCHA, O.L. Measurements of microhardness during transient horizontal directional solidification of Al-Rich Al-Cu alloys: effect of thermal parameters, primary dendrite arm spacing and Al2Cu intermetallic phase. Metals and Materials International, v.21, p.429-439, 2015.

3. BERTELLI, F., BRITO, C., FERREIRA, I.L., REINHART, G., NGUYEN-THI, H., MANGELINCK-NOËL, N., CHEUNG, N., GARCIA, A. Cooling thermal parameters, microstructure, segregation and 
hardness in directionally solidified Al-Sn-(Si;Cu) alloys. Materials in Engineering, v.72, p.3142, 2015.

4. BÖYÜK, U., MARASLI, N., ÇADIRLI, E., KAYA, H., KESLIOGLU, K. Variations of microhardness with solidification parameters and electrical resistivity with temperature for $\mathrm{Al}-\mathrm{Cu}-\mathrm{Ag}$ eutectic alloy. Current Applied Physics, v.12, p.7-10, 2012.

5. ÇADIRLI, E. Effect of solidification parameters on mechanical properties of directionally solidified Al-Rich Al-Cu alloys. Metals and Materials International, v.19, p.411-422, 2013.

6. CARVALHO, D.B., COSTA, T.A., MOREIRA, A.L.S., SILVA, M.A.P.S., DIAS, M., MOUTINHO, D.J.C., ROCHA, O.L. Solidification thermal parameters and dendritic growth during the horizontal directional solidification of Al-7wt.\%Si alloy. Revista Escola de Minas (Impresso), v.67, p.265270, 2014.

7. CARVAlHo, D.B., GUIMARÃES, E.C., MOREIRA, A.L., MOUTINHO, D.J., DIAS FILHO, J.M., ROCHA, O.L. Characterization of the Al-3wt.\%Si alloy in unsteady-state horizontal directional solidification. Materials Research, v.16, p.874-883, 2013.

8. COSTA, T.A., MOREIRA, A.L., MOUTINHO, D.J., DIAS, M., FERREIRA, I.L., SPINELLI, J.E., ROCHA, O.L., GARCIA, A. Growth direction and Si alloying affecting directionally solidified structures of Al-Cu-Si alloys. Materials Science and Technology, v.31, p.1103-1112, 2015

9. DIAS, M., KIKUCHI, R.H., COSTA, T.A., MOREIRA, A.L., ROCHA, O.L. Influência das variáveis térmicas sobre os espaçamentos dendríticos terciários durante a solidificação direcional horizontal da liga Al-6\%Cu. Matéria, v.20, p.47-63, 2015.

10. FERREIRA, I.L., MOUTINHO, D.J., GOMES, L.G., ROCHA, O.L., GOULART, P.R., GARCIA, A. Microstructural development in a ternary $\mathrm{Al}-\mathrm{Cu}-\mathrm{Si}$ alloy during transient solidification. Materials Science Forum, v.636-637, p.643-650, 2010.

11. GARCIA, A. Solidificação Fundamentos e Aplicações. UNICAMP-SP, 2007.

12. GOMES, L.G., Microestrutura Dendrítica, Macrossegregação e Microporosidade na Solidificação de Ligas Ternárias Al-Si-Cu, 2012. Tese de Doutorado - Faculdade de Engenharia Mecânica - Universidade Estadual de Campinas, 2012.

13. GURGEL, R.N. secondary and tertiary dendrite arm spacing relationships in directionally solidified Al-Si alloys. Journal of Materials Science, v.28, pp. 677-683, 1993.

14. MOUTINHO, D.J.C., Análise da Evolução Microestrutural e da Formação de Macrossegregação e Microporosidade na Solidificação Unidirecional Transitória de Ligas Ternárias Al-Cu-Si, 2012. Tese de Doutorado - Faculdade de Engenharia Mecânica Universidade Estadual de Campinas, 2012.

15. NOGUEIRA, M.R., CARVALHO, D.B., MOREIRA, A.L., DIAS FILHO, J.M., ROCHA, O.L. Espaçamentos dendríticos primários da liga $\mathrm{Sn}-5 \% \mathrm{~Pb}$ solidificada direcionalmente em um sistema horizontal. Matéria, v.17, p.1009-1023, 2012.

16. OSÓRIO, W.R., FREITAS, E.S., PEIXOTO, L.C., SPINELLI, J.E., GARCIA, A. The effects of tertiary dendrite arm spacing and segregation on the corrosion behavior of a $\mathrm{Pb} \mathrm{Sb}$ alloy for lead-acid battery components. Journal of Power Sources, v.207, p.183-190, 2012.

17. RANA, R.S., PUROHIT, R., DAS, S. Reviews on the influences of alloying elements on the microstructure and mechanical properties of aluminum alloys and aluminum alloy composites. International Journal of Scientific and Research Publications, v.2, p.1-7, 2012. 
18. ROSA, D.M., SPINELLI, J.E., FERREIRA, I.L., GARCIA, A. Cellular/dendritic transition and microstructure evolution during transient directional solidification of $\mathrm{Pb}-\mathrm{Sb}$ alloys. Metallurgical and Materials Transactions, v.39, pp. 161-2174, 2008.

19. SÁ, F.A., ROCHA, O.L., SIQUEIRA, C.A., GARCIA, A. The effect of solidification variables on tertiary dendrite arm spacing in unsteady-state directional solidification of $\mathrm{Sn}-\mathrm{Pb}$ and $\mathrm{Al}-\mathrm{Cu}$ alloys. Materials Science and Engineering A, v.373, pp. 131-138, 2004.

20. SPINELLI, J.E., SILVA, B.L., GARCIA, A. Assessment of tertiary dendritic growth and its effects on mechanical properties of directionally solidified Sn-0.7Cu-xAg Solder Alloys. Journal of Electronic Materials, v.43, p.1347-1361, 2014.

21. VASCONCELOS, A.J., SILVA, C.V.A., MOREIRA, A.L.S., SILVA, M.A.P.S., ROCHA, O.L. Influence of thermal parameters on the dendritic arm spacing and the microhardness of Al-5.5wt.\%Sn alloy directionally solidified. Revista Escola de Minas, v.67, p.173-179, 2014.

22. VOLLER, V.R. A numerical scheme for solidification of an alloy. Canadian Metallurgical Quarterly, v.37, p.169-177, 1998.

23. YANG, W., YANG, X., JI, S. Melt superheating on the microstructure and mechanical properties of diecast Al-Mg-Si-Mn alloy. Metals and Materials International, v.21, p.382-390, 2015. 